\title{
MATHEMATICAL MODELING OF A HEAT PUMP AND ITS OPERATION MODES
}

\author{
Mehriya Koroli $^{1}$, Oybek Ishnazarov ${ }^{2}$ \\ ${ }^{1}$ Tashkent state technical university named after Islam Karimov, Department of Thermodynamics and Heat Engineering, \\ University - 2, Tashkent, 100095, Uzbekistan \\ ${ }^{2}$ Scientific and Technical Centre of JSC Uzbekenergo, Ashrafiy- 1/9A, Tashkent, 100207, Uzbekistan
}

\begin{abstract}
The paper describes a mathematical model of the heat pump system of autonomous heat supply. Calculations of the temperature at the outlet of the pipeline and the heat pump are given; heat generation and heater condition.
\end{abstract}

\section{Introduction}

The article presents a description of the mathematical model of a heat pump heat supply system operating in conjunction with a vertical ground heat exchanger. The proposed model can be used to simulate systems with different parameters, and identify the most effective of them. The simulation results are presented.

\section{Statement of a question}

The heat pump system for autonomous heat supply of residential and industrial buildings is a variety of heat exchangers utilizing low-potential heats of ambient air, solar energy, water and soil of the surface layers of the Earth, included in a single circuit with the evaporator of the heat pump installation. The advantage of such systems is the availability of a low-grade heat source. Of course, this explains the interest of heat supply enterprises in heat pump installations. The use of heat pump units is one of the most promising areas of energy-saving and environmentally friendly technologies.

When using the heat of the Earth, two types of heat energy can be distinguished - high-potential and lowpotential. The source of high-potential thermal energy is hydrothermal resources - thermal waters heated as a result of geological processes to high temperatures, which allows them to be used for heating buildings. However, the use of the high-potential heat of the Earth is limited to areas with certain geological parameters. Unlike the "direct" use of high-grade heat (hydrothermal resources), the use of low-grade heat of the Earth by means of heat pumps is possible almost everywhere. Currently, this is one of the most dynamically developing areas of the use of non-traditional renewable energy sources.

Thus, the creation of a rational system and its optimization of the parameters of autonomous heat supply, built on renewable energy equipment, is an extremely important stage in their creation, ensuring the best technical and economic characteristics and functioning parameters. This raises the problem of constructing a mathematical model of the system under study. For this, a technique is proposed based on the use of equations describing the features of production, conversion and storage of thermal energy.

\section{System features}

Let us consider the main physical processes occurring in the soil massif of the system for collecting low-potential soil heat (heat collection system) during operation and having a significant impact on the formation of its thermal regime (Fig. 1). During the operational period, the soil mass located within the zone of thermal influence of the register of pipes of the ground heat exchanger, due to seasonal changes in the parameters of the external climate, as well as under the influence of operational loads on the heat collection system, as a rule, is subjected to repeated freezing and thawing. In this case, naturally, there is a change in the state of aggregation of moisture contained in the pores of the soil and in the general case both in the liquid and in the solid and gaseous phases simultaneously.

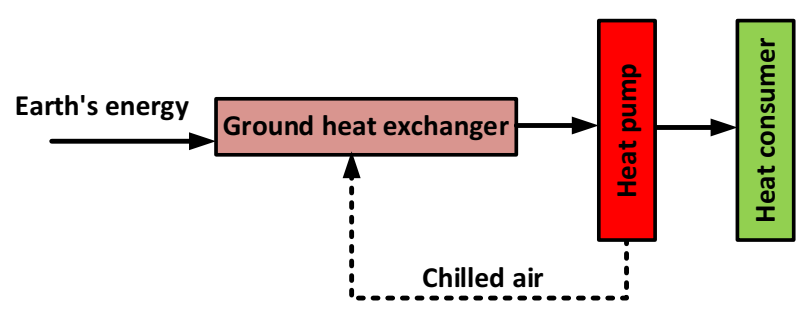

Fig. 1. Energy flows of the Earth.

Thus, the soil massif of the heat collection system, regardless of what state it is in (frozen or thawed), is a complex three-phase polydisperse heterogeneous system, the skeleton of which is formed by a huge amount of solid particles of various shapes and sizes and can be both rigid 
and mobile, depending on whether the particles are firmly bound together, or whether they are separated from each other by matter in the mobile phase. The gaps between solids can be filled with saline moisture, gas, steam and ice, or both. In other words, the medium filling the pore space of a solid skeleton can be in various states of aggregation.

\section{Work overview}

The possibility of using heat pumps in the heating systems of each heat receiving system depends not only on energy and ecology, but also on economic issues. Economic factors relate to efficiency, which depends on the type and condition of the lower sound source, as well as how heat is obtained from the upper source.

In [1], the authors analyzed the energy effects of heat pumps, in which atmospheric air heated in liquid bottles was used as the lower heat source. Energy from the upper source was used to heat the water. In [2], the authors described and analyzed an interacting system of a heat pump (atmospheric air was used as the lower source) with an upper source in which energy was accumulated in the battery. They proposed the concept of "Performance Factor". In [3], the authors developed an innovative system for visualizing and analyzing the efficiency of a heat pump, which uses a ground heat exchanger as the bottom source. A heat pump was used for heating in a prototype greenhouse. Ultimately, the researchers presented synthetic indicators of the thermal regime (operating efficiency, financial ratios), while the energy effects were calculated in terms of limiting the emissions of substances released from the combustion of conventional fuels. In work [4], the authors studied the energy effects of a system in which a heat pump interacted with a solar collector. Energy from the top source was used to heat the liquid in the battery. Researchers have determined performance factors for given system components. They also set the rate of return on finance charges.

In work [5] performed system simulation tests in which the lower heat source consumed energy from the conversion of sunlight in solar air collectors, while the heat pump interacted with a battery filled with a solid subject to phase change. The tests were carried out under laboratory conditions. The author identified the energy effects and pointed out the validity of conducting a thorough economic analysis for this system, which will be used in real structures.

In [6], the authors developed a mathematical model for analyzing the interaction of heat pumps, in which the lower heat source used water collected in a liquid accumulator located in the soil. The results of modeling water temperature variability were determined as a function of the variable temperature of the surrounding soil and the total heat loss from the analyzed accumulator. In [7], the energy effects of a system in which the compressor heat pump interacted with vertical ground heat exchangers were determined. As a result of the analysis, the thermal efficiency of the pipes of the surface heat exchanger and the efficiency factor were determined.

In work [8], the authors analyzed the efficiency of heat pumps interacting with ground heat exchangers. A mathematical model was developed for heat transfer between the ground and the pipelines that make up the lower heat source of the heat exchanger. Studies have shown a high degree of agreement between measured and calculated soil temperatures using the model.

In [9], the authors analyzed the operation of a heat pump, in which the lower heat source was the intake of geothermal water and solar collectors. Using the existing numerical model, they determined the operating parameters of the system under consideration and presented the energy and economic effects, as well as quantitative indicators of reducing emissions of harmful substances into the atmosphere.

The authors of $[10,11]$ analyzed the energy efficiency of the compressor heat pump for laboratory conditions. In work $[8,12]$, the authors analyzed the interaction of a heating system with a heat pump installed in a greenhouse. In addition, the authors $[3,14]$ present the results of an analysis in which a heat pump operating with a heat storage material filled the transition phase. In work [15], the authors developed a system for using heat pumps for heated greenhouses and its effect in comparison with other heat sources. The studies carried out in [14] presented the results of an energy analysis associated with the selection of a lower heat source for operation with a compressor heat pump.

\section{Mathematical equations}

As practice shows, the maximum temperature gradient in the soil mass does not exceed $10 \mathrm{deg} / \mathrm{m}$, and, therefore, the temperature drops at distances measured in millimeters will be very small, as a result of which the heat flux carried by the migrating moisture can be considered negligible.

Thus, without much damage to the accuracy of the mathematical models of the thermal regime of systems for collecting low-potential soil heat, the influence of migration flows of moisture in the pore space of the soil mass on the processes of heat and mass transfer can be neglected.

In fig. 2 shows a simplified diagram of a heat pump. In accordance with fig. 2 relations between the parameters of energy flows are represented:

$$
Q_{k}=Q_{0}+Q_{e l}, \varepsilon=Q_{k} / Q_{e l}
$$

From the above ratios, it follows that the amount of heat generated by the heat pump $Q_{k}$ is equal to the sum of the amount of heat taken in the evaporator from the natural source $Q_{0}$ and the thermal equivalent of electrical energy consumed to drive the compressor $Q_{e l}$. The ratio $Q_{k} / Q_{e l}$, is the transformation ratio of low-grade heat or the conversion ratio of the heat pump $\varepsilon$, which characterizes the energy efficiency of its operation. 


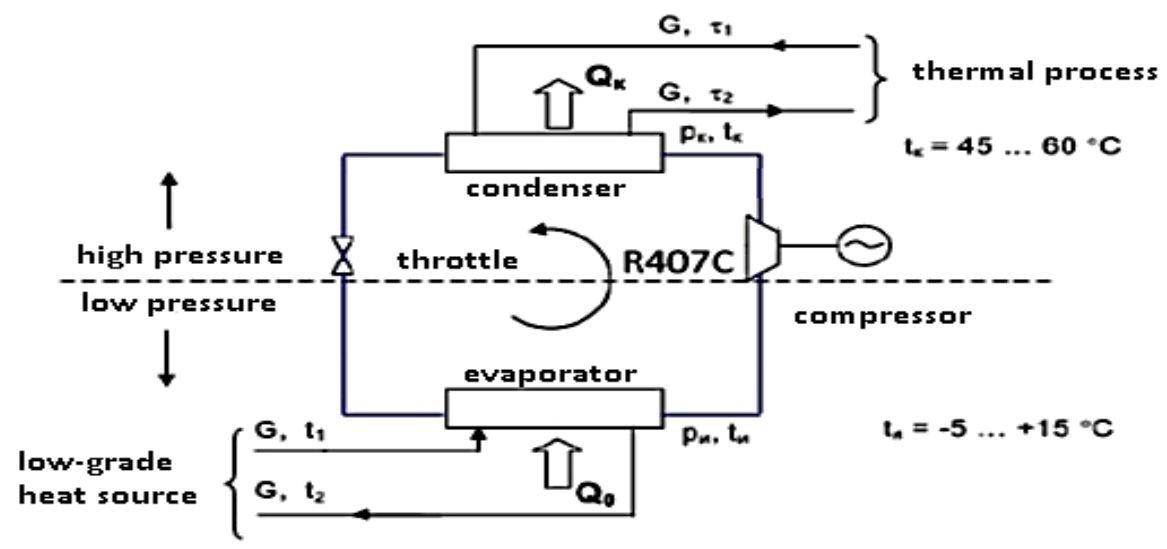

Fig. 2. Simplified diagram of a heat pump.

The technological regime in the soil layers is characterized by: the ratio of the heat transfer of the working fluid in the pipes and the heat loss to the environment. In this case, we will use the heat conduction equation, which characterizes the process under study to the required extent:

$$
\left(\frac{\partial^{2} T}{\partial x^{2}}+\frac{\partial^{2} T}{\partial y^{2}}+\frac{\partial^{2} T}{\partial z^{2}}\right)+\frac{q_{v}}{\lambda}=\frac{1}{a} \frac{\partial T}{\partial t}
$$

where $q_{v}$ is heat source member, $\mathrm{W} / \mathrm{m}^{3} ; \lambda$ - soil conductivity coefficient, $\mathrm{W} / \mathrm{mK} ; T$ is temperature in the soil; $x, y$ and $z$ are space variables.

The heat transfer coefficient for ground heat transfer $\left(U_{g}\right)$ is determined according to the equation [12]

$$
U_{g}=0.999\left(\frac{k_{g}}{d_{g}-d_{p}}\right)+1.37\left(\frac{k_{g} P p}{A_{p}}\right)
$$

where $k_{g}$ is the thermal conductivity of the ground, $d_{g}$ is the depth to the water table or the constant source/sink from the ground surface, $d_{p}$ is the pond depth, and $\mathrm{P}_{\mathrm{p}}$ is the pond perimeter. The conduction heat transfer between the ground and the pond is then given by

$$
q=U_{g} A_{p}\left(T_{g}-T_{p}\right),
$$

where $A_{p}$ is pond area, $\mathrm{m}^{2} ; T_{g}$ is ground temperature ${ }^{\circ} \mathrm{C}$; $T_{p}$ is pound temperature ${ }^{\circ} \mathrm{C}$.

According to [13] the heat pump can be represented as two sections of a continuous flow heat exchanger, separated by a wall with internal intercooling and simultaneous heating. The heat transferred from the cold section $Q_{x}$ and the heat transferred to the heated medium $Q_{g}$ are related to the power to drive the compressor $P_{t n}$ of the heat pump, by the equation. The heat balance equation for heat fluxes in a heat pump by

$$
M_{X} C_{B}\left(t_{x 2}-t_{x 1}\right)-Q_{x}=m_{t x} C_{B} \frac{d t_{x 1}}{d \tau}
$$

$$
Q_{g}+M_{\mathrm{H}} C_{B}\left(t_{\mathrm{H} 2}-t_{\mathrm{H} 1}\right)=m_{\mathrm{TH}} C_{B} \frac{d t_{\mathrm{H} 2}}{d \tau} .
$$

The power to drive the compressor of the heat pump is variable, and the transformation ratio of the heat pump will change due to changes in temperatures in the condenser and evaporator.

\section{Simulation results}

The above equations show the main results. It should be noted that it is necessary to dig the ground to lay the pipes, the initial cost of installing the soil heat recovery system is higher than when using traditional heating methods. It is important to evaluate the various heat collector schemes in order to get the most out of your ground source heat pump installation. There are a number of factors that will affect the efficiency of a heat exchanger, including:

- Variations in soil properties;

- Pipe diameter;

- Thermal conductivity of the pipe;

- Liquid consumption;

- Thermal load of the system;

- Weather conditions of the area.

Table 1 shows the average monthly minimum and maximum temperature values for the city of Tashkent.

Fig. 3-5 show the results of simulation modeling of the heat pump operating modes. In fig. 3 shows a histogram of the outlet temperature showing the outlet temperature of the pipeline (a) and the heat pump (b) as a function of time. In fig. 4 is a graph of heat production showing the heat production of the heat pump over time. In fig. 5 is a graph of the status of the heater, which shows the operation of the heat pump.

Table 1. Average monthly minimum and maximum temperature values for the city of Tashkent

\begin{tabular}{|c|c|c|}
\hline Month & $\begin{array}{c}\text { Average - month minimum, } \\
\mathbf{0}_{\mathbf{C}}\end{array}$ & Average - month maximum, ${ }^{\mathbf{0}} \mathbf{C}$ \\
\hline January & -1.5 & 6.9 \\
\hline February & 0 & 9.4 \\
\hline March & 4.8 & 15.2 \\
\hline April & 9.8 & 22 \\
\hline May & 13.7 & 27.5 \\
\hline
\end{tabular}




\begin{tabular}{|c|c|c|}
\hline Jun & 18.1 & 33.4 \\
\hline July & 19.7 & 35.6 \\
\hline August & 18.1 & 34.7 \\
\hline September & 13 & 29.3 \\
\hline October & 7.8 & 21.8 \\
\hline November & 4.1 & 14.9 \\
\hline December & 0 & 8.8 \\
\hline
\end{tabular}

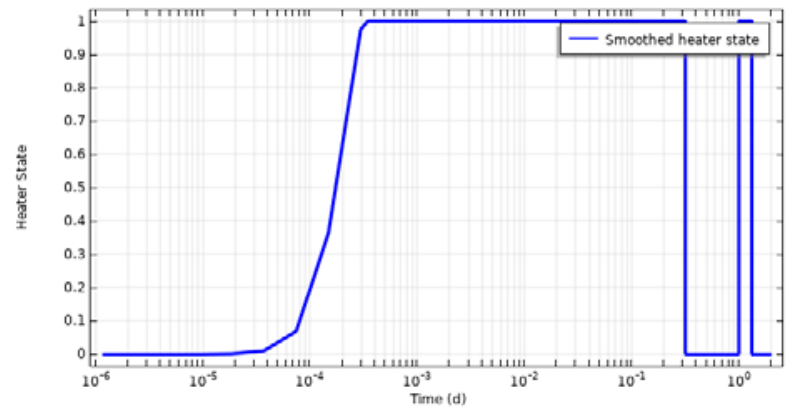

a)

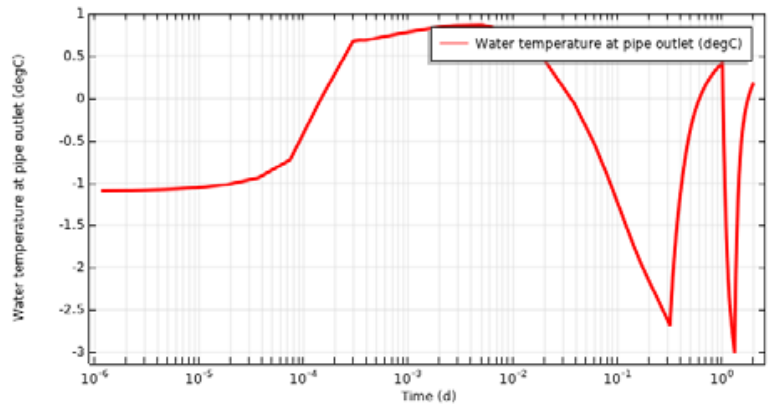

b)

Fig. 3. The outlet temperature histogram displays the temperature at the outlet of the pipeline (a) and the heat pump (b)

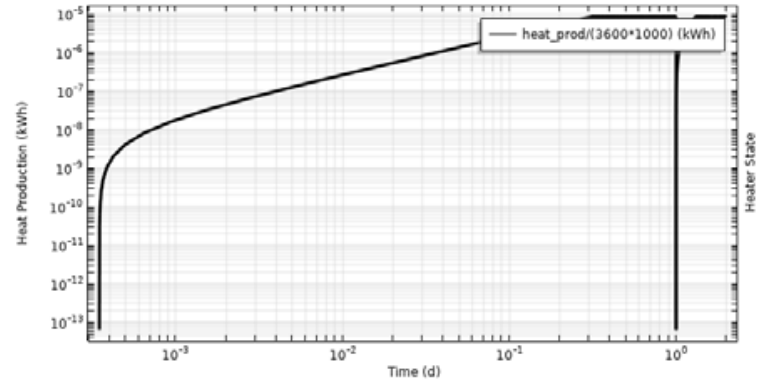

Fig. 4. Heat generation

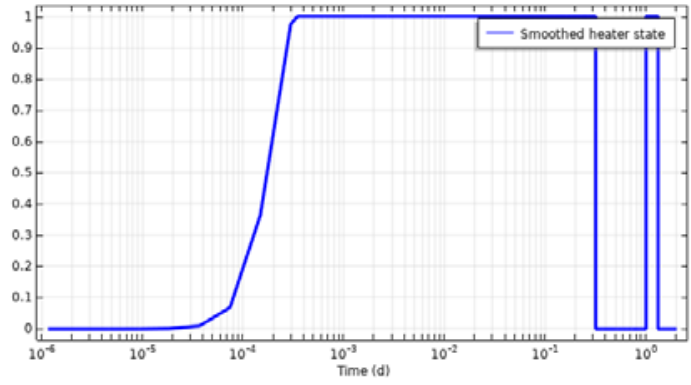

Fig. 5. State of the heat pump.

\section{Conclusion}

Thus, a heating system with a heat pump has a good potential for energy saving in heating systems such as dehumidification, water heating and space heating. The presented equations make it possible to determine the operating mode of the heat pump. Advanced heat pump controls are recommended to improve performance

\section{References}

1. Xu G., Zhang A., Deng S. 2006. simulation study on the operating performance of a solar-air source heat pump water heater. Applied Thermal Engineering, 26 (11-12), p. $1257-1265$.

2. Kaygusuz K. 1995. Performance of solar- assisted heat pump systems. Applied Energy, 51(2), p. 93-109.
3. Nagano K., Katsura T., Takeda S. 2006. Development of a design and performance prediction tool for the ground source heat pump system. Applied Thermal Engineering, 26(14-15), p. 1578-1592.

4. Hawlader M.N.A., Chou S.K., Ullah M.Z. 2001. The performance of a solar assisted heat pump water heating system. Applied Thermal Engineering, 21(10), p. 10491065 .

5. Kaygusuz K., Ayhan T. 1999. Experimental and theoretical investigation of combined solar heat pump system for residential heating. Energy Conversion and Management, 40(13), p. 1377-1396.

6. Yumruta R., Unsal M. 2000. A computational model of a heat pump system with a hemispherical surface tank as a ground heat source. Energy, 25(4), p. 371-388.

7. Hepbasli A., Akdemir O., Hancioglu E. 2003. Experimental study of a closed loop vertical ground heat pump system. Energy Conversion and Management, 42(4), p. 527-548.

8. Esen H., Intali, Esen M. 2006. Numerical and experimental analysis of a horizontal ground-coupled heat 
pump system. Building and Environment, 42(3), p. 11261134.

9. Trillat-Berdal V., Souyri B., Fraisse G. 2006. Experimental study of a ground-coupled heat pump combined with thermal solar collectors. Energy and Buildings, 28(12), p. 1477-1484.

10. Knaga J. 2007. Changeability of heat output of heat pump with scroll type compressor, TEKA Commission of Motorization and Power Industry in Agriculture VIIA, p. 41-46.

11. Knaga J. 2008. Energy effi ciency of small compressor assisted air-water type heat pumps, TEKA Commission of Motorization and Power Industry in Agriculture VIII 2008, p. 99-106.

12. Benli H, Durmus A. 2009. Evaluation of groundsource heat pump combined latent heat storage system performance in greenhouse heating. Energy Buildings 41, p. $220-228$.

13. Benli H. 2011. Energetic performance analysis of a ground-source heat pump system with latent heat storage for a greenhouse heating Energy Conversion and Management 52, p. 581-589.

14. Kurpaska S. 2007. Energy analysis for lower heat sources of heating pump while heating a plastic tunnel. Inynieria Rolnicza, 9(97), p. 103-111.

15. Katsunori N, Takao K, Sayaka T. 2006. Development of a design and performance prediction tool for the ground-source heat pump system. Applied Thermal Engineering 26, p. 1578-1592.

16. Andrew D Advances in Modeling of Ground-Source Heat Pump Systems Master of Applied Science University of Windsor (Ontario: Windsor) 1992, p. 155 17. Slesarenko I Solar water heater systems evolution analysis .Mining information and analytical bulletin (scientific and technical journal) 2015 .S36 178-86. 